\title{
Transmit Array Antenna Using Nonuniform Dielectric Layer
}

\author{
Mojtaba Moeini-Fard, Mohammad Khalaj-Amirhosseini \\ School of Electrical Engineering, Iran University of Science and Technology, Tehran, Iran \\ Email address: \\ mmoeinifard2000@yahoo.com (M. Moeini-Fard), Khalaja@iust.ac.ir (M. Khalaj-Amirhosseini)
}

\section{To cite this article:}

Mojtaba Moeini-Fard, Mohammad Khalaj-Amirhosseini. Transmit Array Antenna Using Nonuniform Dielectric Layer. Advances in Wireless Communications and Networks. Vol. 3, No. 3, 2017, pp. 23-28. doi: 10.11648/j.awcn.20170303.11

Received: May 13, 2017; Accepted: May 25, 2017; Published: June 30, 2017

\begin{abstract}
A shaped-beam Transmit Array Antenna based on nonuniform dielectric layer is proposed. Stepping the surface of dielectric layer provides the required phase shift at each point on the surface of transmit array antenna to produce a squared cosecant beam on the other side. In order to analyze this kind of antennas, a simple method based on an equivalent circuit model is introduced, in which the transmission coefficient of each element for oblique incident field is obtained. The design process consists of two steps. First, a phase-only technique is applied to obtain the required phase-shift distribution on the surface of transmit array antenna which generates the shaped pattern. The second stage consists of determining the thickness of each element to achieve the phase distribution obtained in the previous step. Using this method a shaped beam transmit array antenna in the 9.5-10.5 GHz band is designed, manufactured and also validated by the Computer Simulation Technology (CST) Microwave Studio TM package. The results of test in an anechoic chamber, shows a good agreement between measured, simulated and theoretical radiation patterns.
\end{abstract}

Keywords: Nonuniform Dielectric Layer, Transmit Array Antenna, Phase Response, Lens Antenna

\section{Introduction}

Transmit Array Antennas have been considered as lowprofile and low-cost planar alternatives to dielectric lens antennas for millimeter wave applications like radars, electronic beam-forming systems, imaging systems, and quasi-optical power combiners [1-6]. These type of antennas are planar like Fresnel [7-8] and two-layer ones [9]. Transmit array antennas are based on similar concepts as for reflect array antennas [10-12] except that they operate in a transmission mode rather than in reflection. Contrary to the reflect array antennas, such configurations offer several advantages, such as reduction of blockage effects due to the focal source. In transmit array antennas, there is no completely reflective ground plane, so that the incident electromagnetic wave can be transmitted through the surface and converted from spherical wave into a plane wave. Like a reflect array antenna, the elements of a transmit array antennas produce specific phase shift that when properly tuned, can focus incident waves. So, the two most important design criteria for an element of transmit array antenna is its phase range and insertion loss. Firstly, in order for an element design to be suitable for an arbitrarily large array where there is possibly phase wrapping in the aperture fields, a phase tuning range of $360^{\circ}$ is required. Secondly, the insertion loss must be minimized. Furthermore, to achieve proper beam forming with good control of side-lobe levels, the variation in the element's insertion loss over the entire phase tuning range should also be small. Almost all elements presented in the literature are based on microstrip patches [1-6]. However, the microstrip patch antennas are inherently narrow-band radiating elements and the mutual couplings between microstrip elements printed on standard substrates are significant; in addition, there are conductor and surface wave losses that should be noticed. Here, we have taken a rather new approach to reduce all mentioned shortcomings. The idea which has been presented by the authors for reflect array antennas [11], is to construct a nonuniform dielectric layer as a transmit array antenna to produce the desired shaped radiation beam. The dielectric layer with variable thickness provides the required phase shift at each point on the surface of transmit array antenna to produce a squared cosecant beam. Stepping dielectric layer with variable thicknesses provides this nonuniform formation. Absence of the resonator elements in proposed structure will reduce the dielectric and conductor losses, which are the main losses in transmit array antennas at resonance frequency. Moreover, a simple method is introduced for designing and analyzing 
such transmit array antenna. This method is based on the transmission line model. So, in the first step, the phase response of an infinite dielectric layer versus its thickness variation is plotted and modeled with this equivalent circuit and compared with the full-wave electromagnetic simulation results. Then, by implementing this model, the proper thickness of each element is calculated. Finally, to demonstrate the usefulness of the proposed antenna, a sample antenna is designed, fabricated and tested and also validated by the Computer Simulation Technology (CST) Microwave Studio TM package, which utilizes the finite integration technique for electromagnetic computation.

\section{Analysis of Nonuniform Dielectric Layer}

Figure 1 shows the proposed antenna, in which a feed antenna illuminates a nonuniform dielectric layer with dielectric constant $\varepsilon_{r}$ and dimensions $L_{x}, L_{y}$ and $h(x, y)$. The incident electric fields of the feed antenna arrive at different points on the dielectric surface with different phases, with respect to different paths. By considering the thickness of dielectric layer, these fields will transfer with different transmission coefficients. If the thickness pattern of dielectric layer are selected appropriately such that the phase of the transferred fields are equal in a special direction, then this aperture field produces high gain radiation pattern. On the other hand, the nonuniform dielectric layer with variable thickness is used to control the transmission phases. The critical feature of implementing such antennas is how the thickness of the individual element is selected to shift the incident wave with the desired phase. In practice, to implement such nonuniform dielectric layer, it must be subdivided into small uniform elements with dimension $d_{x} \times d_{y} \times h_{m n}$ as shown in Figure 1. The proposed antenna is similar to a planar transmit array antenna with $M$ by $N$ dielectric elements, where $M=L_{x} / d_{x}$ and $N=L_{y} / d_{y}$.

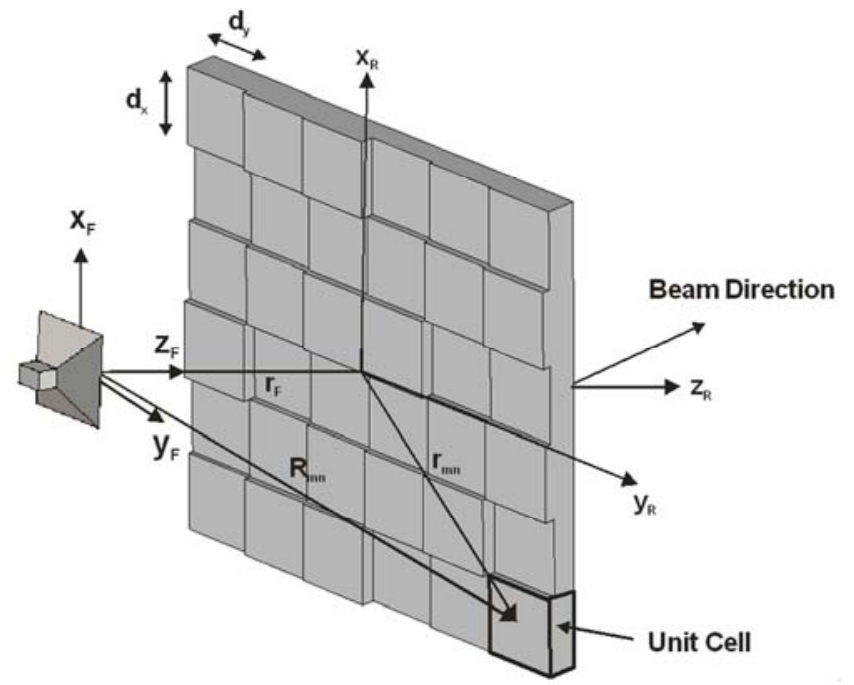

Figure 1. Total view of nonuniform transmit array antenna and its coordinate systems.
Figure 2(a) shows a typical element with thickness of $h_{m n}$. As depicted in this figure, two different polarizations are possible, one $T M$ and other $T E$ due to the incident wave polarization.

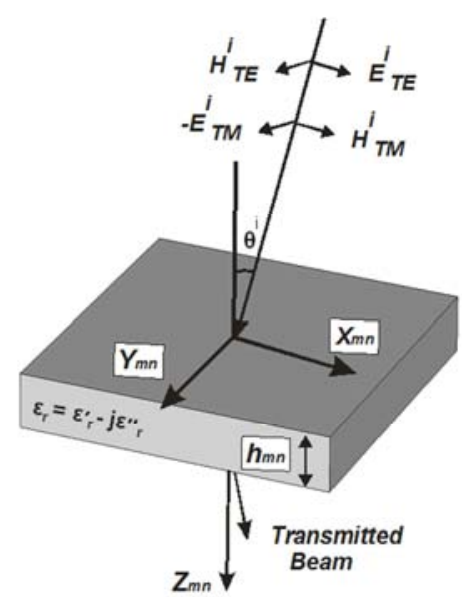

(a)

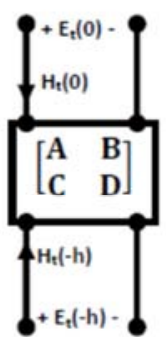

(b)
Figure 2. Single element configuration with its coordinate system (a) and its equivalent two port circuit model (b).

The incident field $\left(E^{i}\right)$ propagates obliquely in $(X Z)_{m n}$ plane of the $m n$-th element coordinate system, with an angle of incident $\theta^{i}$. By assuming each element as a homogeneous planar layer, two port circuits are obtained for elements with well-known transmission line $A B C D$ matrices (as shown in Figure 2(b)). The transverse electric and magnetic fields on the surfaces $z=0$ and $z=h_{m n}$ are related to each other by the $A B C D$ matrix as follows:

$$
\left[\begin{array}{l}
E_{t}(0) \\
H_{t}(0)
\end{array}\right]=\left[\begin{array}{ll}
A_{m n} & B_{m n} \\
C_{m n} & D_{m n}
\end{array}\right]\left[\begin{array}{l}
E_{t}\left(h_{m n}\right) \\
H_{t}\left(h_{m n}\right)
\end{array}\right]
$$

where

$$
\begin{aligned}
& E_{t}(z) \triangleq\left\{\begin{array}{c}
E_{x}(z), T E \\
E_{y}(z), T M
\end{array}\right. \\
& H_{t}(z) \triangleq\left\{\begin{array}{c}
H_{y}(z), T E \\
-H_{x}(z), T M
\end{array}\right.
\end{aligned}
$$

are the transverse electric and magnetic fields. The $A B C D$ parameters of $m n$-th cell is defined with the characteristic impedance $\left(Z_{c}\right)$ and propagation constant $(\beta)$ of a dielectric layer in a waveguide as follows [1]:

$$
\begin{gathered}
A_{m n}=D_{m n}=\cos \left(\beta h_{m n}\right) \\
B_{m n}=Z_{c}^{2} C_{m n}=j Z_{c} \sin \left(\beta h_{m n}\right)
\end{gathered}
$$

where

$$
\begin{gathered}
Z_{c}=\left\{\begin{array}{c}
\frac{\sqrt{\mu_{0} / \varepsilon_{0}}}{\sqrt{\varepsilon_{r}-\sin ^{2}\left(\theta^{i}\right)}}, T E \\
\frac{\sqrt{\mu_{0} / \varepsilon_{0}}}{\varepsilon_{r}} \sqrt{\varepsilon_{r}-\sin ^{2}\left(\theta^{i}\right)}, T M
\end{array}\right. \\
\beta=\frac{\omega}{c} \sqrt{\varepsilon_{r}-\sin ^{2}\left(\theta^{i}\right)}
\end{gathered}
$$


where $c$ is the velocity of the light and $\omega$ is the angular frequency. Furthermore, there are two boundary conditions as follows:

$$
\begin{aligned}
& \qquad E_{t}(0)=\left\{\begin{array}{c}
-Z_{s} H_{t}(0)+2 E^{i}, T E \\
-Z_{s} H_{t}(0)+2 \cos \left(\theta^{i}\right) E^{i}, T M
\end{array}\right. \\
& \qquad E_{t}\left(h_{m n}\right)=Z_{L} H_{t}\left(h_{m n}\right) \\
& \qquad \mathrm{T}_{\mathrm{mn}}=\left\{\begin{array}{c}
E_{t} \\
E_{t}\left(h_{m n}\right)
\end{array}\right. \\
& \text { The transmitted field on each element is com } \\
& \text { multiplying the transmission coefficient }(\mathrm{T} \text { mn }) \text { to } \\
& \text { field. Modeling this electric field as an apert } \\
& \text { distribution, the far field pattern can be obtain } \\
& \text { According to relations }(4)-(11), \text { the phase and mag } \\
& \text { transmission coefficient is obtained as follows } \\
& \qquad \phi_{\mathrm{T}_{\mathrm{mn}}}=-\tan ^{-1}\left(0.5\left(\frac{Z_{s}}{Z_{c}}+\frac{Z_{c}}{Z_{s}}\right) \tan \left(\beta h_{m n}\right)\right) \\
& \qquad\left|\mathrm{T}_{\mathrm{mn}}\right|=\frac{2}{\sqrt{4 \cos ^{2}\left(\beta h_{m n}\right)+\left(\frac{Z_{s}}{Z_{c}}+\frac{Z_{c}}{Z_{s}}\right)^{2} \sin ^{2}\left(\beta h_{m n}\right)}}
\end{aligned}
$$$$
\mathrm{T}_{\mathrm{mn}}=\left\{\begin{array}{c}
E_{t}\left(h_{m n}\right) / E^{i}, T E \\
E_{t}\left(h_{m n}\right) /\left(E^{i} \cos \left(\theta^{i}\right)\right), T M
\end{array}=\frac{2 Z_{L}}{\left(A+Z_{s} C\right) Z_{L}+\left(B+Z_{s} D\right)}\right.
$$

The transmitted field on each element is computed by multiplying the transmission coefficient $\left(\mathrm{T}_{\mathrm{mn}}\right)$ to incident field. Modeling this electric field as an aperture field distribution, the far field pattern can be obtained [13]. According to relations (4)-(11), the phase and magnitude of where

$$
Z_{L}=Z_{s}=\left\{\begin{array}{l}
\sqrt{\mu_{0} / \varepsilon_{0}} / \cos \left(\theta^{i}\right), T E \\
\sqrt{\mu_{0} / \varepsilon_{0}} \cos \left(\theta^{i}\right), T M
\end{array}\right.
$$

The transmission coefficient of $m n$-th element will be determined using (1), (8) and (9) as follows:
It is seen from (12) and (13) that the magnitude and phase of transmission coefficient are dependent on the thickness and electric permittivity of the element.

The magnitude of transmission coefficient is less than 1.0 and tends to 1.0 as $Z_{s}$ tends to $Z_{c}$ or equivalently as $\varepsilon_{r}$ approaches 1.0. Therefore, we should choose $\varepsilon_{r}$ as low as possible.

It is notable that the phase of transmission coefficient of each element has to cover $360^{\circ}$ with variation of its thickness $h_{m n}$ in the practical range. This fact is demonstrated in the Figure 3, which shows a typical phase shift of an element with respect to its thickness $h_{m n}$ for normal incident with dielectric constant $\varepsilon_{r}=3$ and at operating frequency of $10 \mathrm{GHz}$. It is evident from Eq. (11) that for normal incident, which is used in the design process of transmit array antennas, the transmission coefficient for $T E$ and $T M$ modes are identical. Therefore, the phase of the transmitted field shown in Figure 3 as the presented method, corresponds to both $T E$ and TM modes. So in the Figure 3 just one phase curve of the transmission coefficient is plotted for the presented method result. Also, Figure 3 shows that the theoretical result agree well with the result obtained by CST simulation, in which a simple dielectric element with variable thickness is simulated with periodic boundary conditions.

It must be noted that, the nonuniformity of the transmit array's surface was not accounted in the analysis of the element. This assumption would not involve a significant error at the final results because of the smooth variation of the thickness in comparison with wavelength.

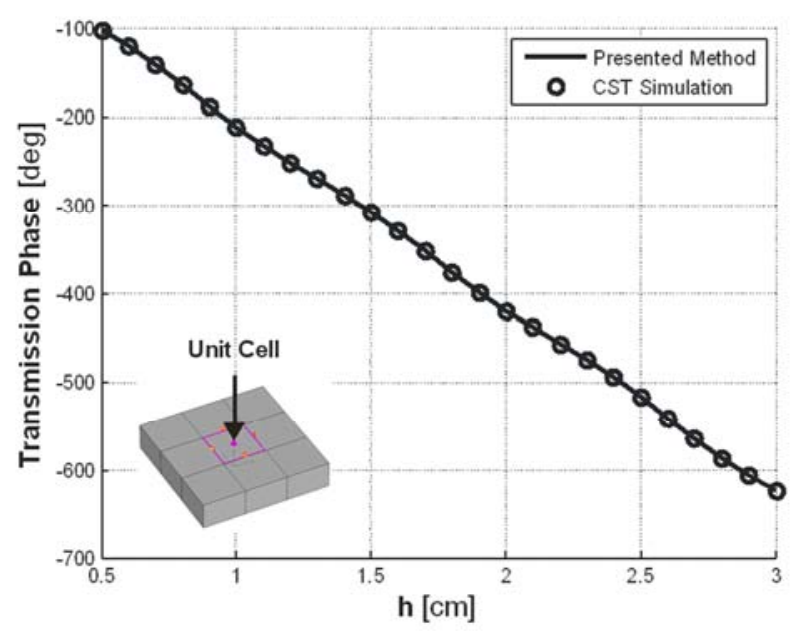

Figure 3. Phase of the transmitted field for normal incident versus thickness.

\section{Design of Nonuniform Elements}

In this section, the designing approach of the nonuniform transmit array antenna is introduced. For the design of a desired beam transmit-array, a phase-only synthesis is applied to compute the phase of the transmitted field at each transmit-array element. Then, the thicknesses of the dielectric elements are adjusted to implement the required phase distribution. The amplitude of the field on the transmit-array surface is imposed by the radiation pattern of the feed, assuming that the transmit-array elements are in the far field region of the feed source. Then, only the phase can be adjusted to shape the beam, which means that a phased-only pattern synthesis technique must be applied. A linearly polarized horn antenna with small aperture size of $2.38 \times$ $2.4 \mathrm{~cm}^{2}$ has been used to feed the transmit-array, and the far field has been modeled as a $\cos ^{q}$ function in order to determine the illumination level on the transmit-array surface. The $q$ value has been calculated to match the radiation pattern of the horn at the design frequency (10 $\mathrm{GHz}$ ). The phase distribution that will be used to produce the squared cosecant beam is obtained by using an analytic phase-only synthesis technique, as described in [14]. As shown in Figure 1, the transmitted field from the transmitarray in the $Z>0$ region, will be in the form of

$$
E\left(\hat{u}_{0}\right)=\sum_{m=1}^{M} \sum_{n=1}^{N} F\left(\vec{r}_{m n} \cdot \vec{r}_{f}\right) \cdot\left|\mathrm{T}_{\mathrm{mn}}\right| \cdot \exp \left\{-j k_{0}\left|\vec{r}_{m n}-\vec{r}_{f}\right|+j \phi_{\mathrm{T}_{m n}}\right\}
$$


where $F$ is the feed pattern function, $\left|\mathrm{T}_{\mathrm{mn}}\right|$ and $\phi_{\mathrm{T}_{m n}}$ are magnitude and phase of transmission coefficient at the $m n$-th element position. Terms $\vec{r}_{m n}$ and $\vec{r}_{f}$ are the position vectors of the $m n$-th element and the feed horn antenna, respectively. The condition for an array aperture distribution to be in phase with the phase distribution of the squared cosecant beam, is given by

$$
\phi_{\mathrm{T}_{m n}}=\phi_{m n}+k_{0} R_{m n}+2 p \pi, p=0, \pm 1, \pm 2
$$

where $R_{m n}$ is the distance from the feed source to center of surface of the $m n$-th array element, i.e., $R_{m n}=\left|\vec{r}_{m n}-\vec{r}_{f}\right|$, and is slightly dependent on the thickness of the $m n$-th element, $h_{m n}$. Also, phase $\phi_{m n}$ is the required phase of the $m n$-th element obtained from the analytic phase-only synthesis technique [14]. According to the relation (12), the proper thickness of the $m n$-th element $\left(h_{m n}\right)$ is obtained as follows

$$
h_{m n}=\frac{1}{\beta} \tan ^{-1}\left(\frac{z_{s}}{Z_{c}} \tan \left(-\phi_{\mathrm{T}_{m n}}\right)\right)
$$

It should be mentioned that the element dimensions $d_{x} \times d_{y}$ are important factors in the final results. The required phase $\phi_{\mathrm{T}_{m n}}$ of $m n$-th element is determined by the relation (15) at the center of the element. This causes a significant phase error, which will increase the output side lobe levels. In order to avoid this phase error, the elements size must be adjusted. Simulation of different element sizes shows that selecting $d_{x}=d_{y}=\lambda / 2$ is a suitable choice in constructing nonuniform transmit array antenna at operating frequency of $10 \mathrm{GHz}$.

\section{Measurement and Validation}

In this section, the performance of the shaped beam transmit array antenna is verified through designing and fabrication of a nonuniform transmit array antenna for the purpose of having a squared cosecant radiation beam at frequency of $10 \mathrm{GHz}$. The desired antenna was designed using aforesaid theory and then fabricated as shown in Figure 4. The nonuniform dielectric layer is formed by machining a dielectric layer of ERTALON (6PLA) with the dielectric constant $\varepsilon_{r}=3$ and dissipation factor 0.016 . We consider a $L=10 \lambda_{0}=30 \mathrm{~cm}$ square nonuniform transmit array antenna in order to have a squared cosecant beam. The dielectric layer is subdivided into 400 elements of equal size of $d_{x}=d_{y}=\frac{\lambda}{2}=1.5 \mathrm{~cm}$ arranged in a lattice of $20 \times 20$ elements. A linearly polarized horn antenna with small aperture size of $2.38 \times 2.4 \mathrm{~cm}^{2}$ is used as a feed of transmit array antenna to minimize the feed-blockage effect. The feed horn $3 \mathrm{~dB}$ beam width is $64.3^{\circ}$ for H-plane pattern and $57.2^{\circ}$ for E-plane pattern at $10 \mathrm{GHz}$ and its phase center located at $r_{f}=L=28 \mathrm{~cm}$ to maximize the antenna efficiency. First, it is interesting to know how much the phase diagram changes with frequency. Actually, more frequency bandwidth occurs when the phase curve of cells have linear variation versus frequency with suitable slope depending on their location.
Figure 5 shows the variation of phase curves for different frequencies centered at $10 \mathrm{GHz}$.

These phase curves are obtained by the normal incident wave assumption. But this is valid for the central elements of the centered fed transmit array antenna, which is for angles not too far from the normal incident. As the largest fraction of the incident power is transmitted by the central part of transmit array antenna, the normal incident assumption can provide good predictions for central fed transmit arrays with reasonably large $F / D$.

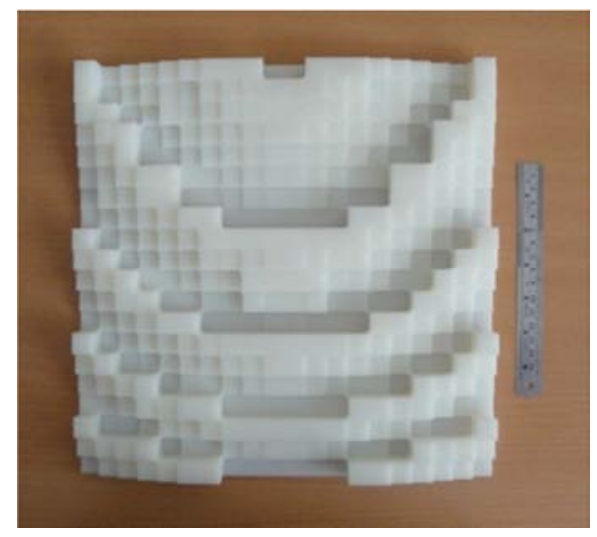

Figure 4. Fabricated nonuniform dielectric transmit array antenna.

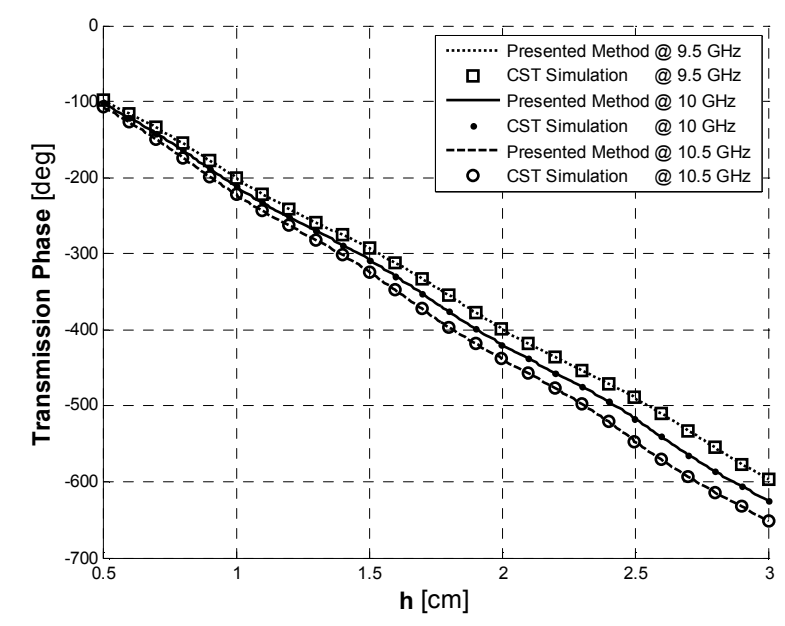

Figure 5. Curves of the transmission phase for different frequencies.

To show the effect of incident angle, different phase shift curves with respect to different incident angles are plotted in Figure 6. It is clear that, the maximum phase shift difference between normal incident and incident of $28^{\circ}$, which is respect to edge elements angle, is about $26.9^{\circ}$. Therefore, in this structure, the normal incident assumption would not impose a significant error at the designing process.

Figure 7 shows the required phase shift $\left(\phi_{\mathrm{T} m n}[\mathrm{deg}]\right)$ of each element and the corresponding dielectric thickness $\left(h_{m n}\right)$. It consists of uniform elements with different thicknesses in the range of $0.5-3 \mathrm{~cm}$. One can see from (6), (7), (10) and (13) that the magnitude of transmission coefficients vary slightly between 0.867 to 0.872 for normal incidence on cells with thickness between $0.5-3 \mathrm{~cm}$, so it is nearly constant. 


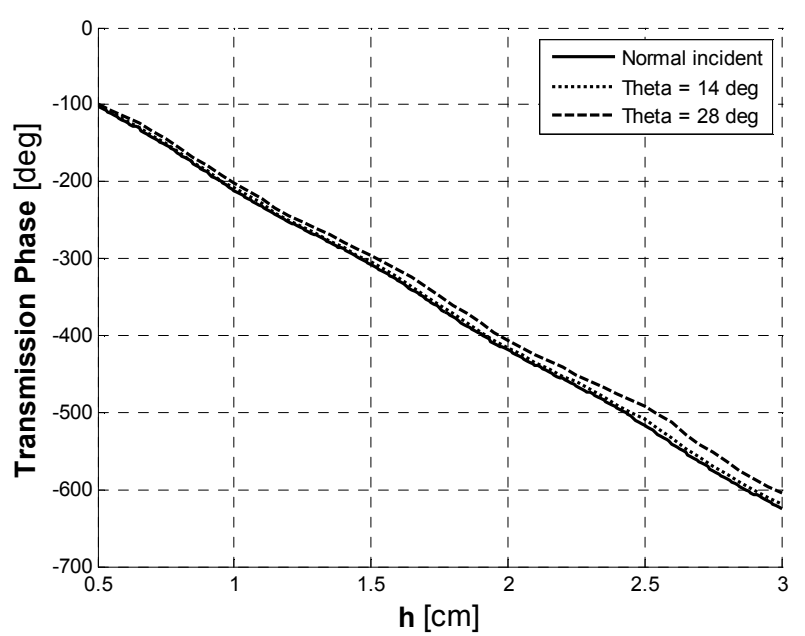

Figure 6. Curves of the transmission phase for different values of $\theta^{i}$ incident angle.

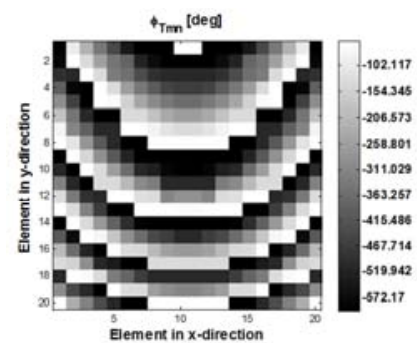

(a)

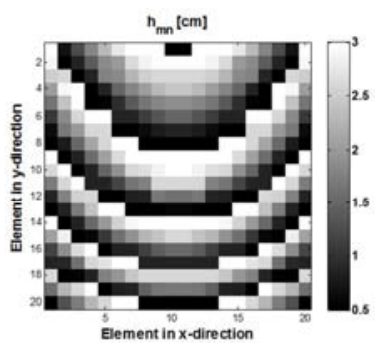

(b)
Figure 7. (a) The required phase shifts of elements and (b) corresponding $h_{m n}$ values for squared cosecant beam transmit array antenna.

Designed Antenna was simulated by CST Microwave Studio (MWS) and the results are compared with theoretical results. Also, the fabricated antenna has been tested in a compact anechoic chamber of $7.2 \mathrm{~m}$ long $\times 3.6 \mathrm{~m}$ wide $\times 3.6 \mathrm{~m}$ high in the frequency range of $30 \mathrm{MHz}$ to $18 \mathrm{GHz}$. Figure 8 shows the measured co-polar and cross-polar radiation patterns, at the central frequency of $10 \mathrm{GHz}$, compared with the simulation and theoretical patterns. Although the measured main lobe is in good agreement with simulation and theoretical predictions, two side lobes appear at $13.8^{\circ}$ and $37.2^{\circ}$. As the transmit array structure is sensitive to thickness of the elements, these side lobes are probably caused by the thickness tolerances of fabrication. Also, the tolerances in the nominal value of the dielectric permittivity can be attributed to these discrepancies. The low cross-polar components of measurement are also plotted with $-17.6 \mathrm{~dB}$ below the peak gain. One may conclude that the existing cross-polarization in the measurement, unlike the simulation and theoretical results with no cross, is attributed to the scattering from the supporting metal bars of antenna. Similar measured patterns are found in the $10 \%$ bandwidth from $9.5-10.5 \mathrm{GHz}$. The measured, simulated and theoretical patterns at $9.5 \mathrm{GHz}$ and $10.5 \mathrm{GHz}$ are shown in Figures 9 and 10, respectively. For these measured patterns, the squared cosecant shapes are conserved but a dip appear in the middle of the band. This discrepancy is mainly produced by the phase center variation of the feed horn antenna with respect to frequency, which caused some phase error on the surface of the transmit array antenna.

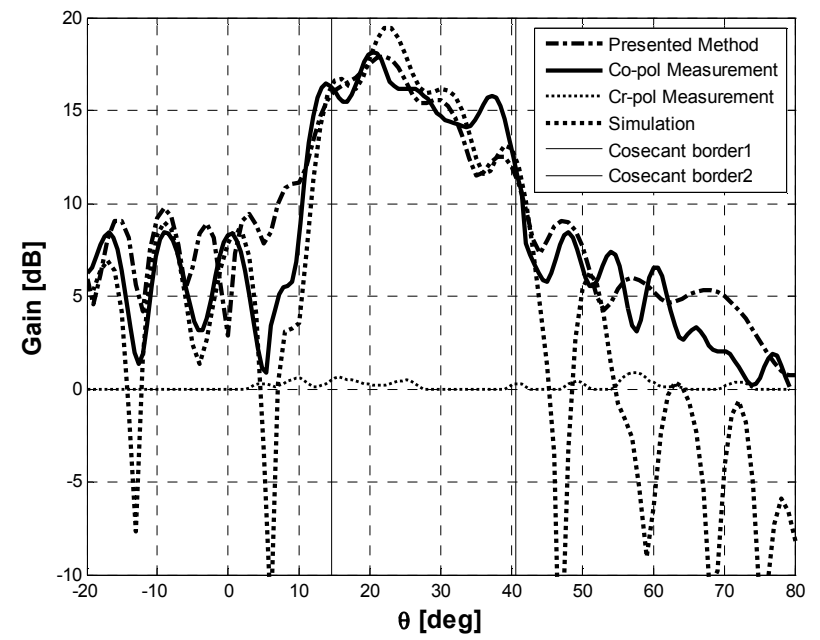

Figure 8. Comparison between measured, simulated and theoretical radiation patterns at central frequency $10 \mathrm{GHz}$.

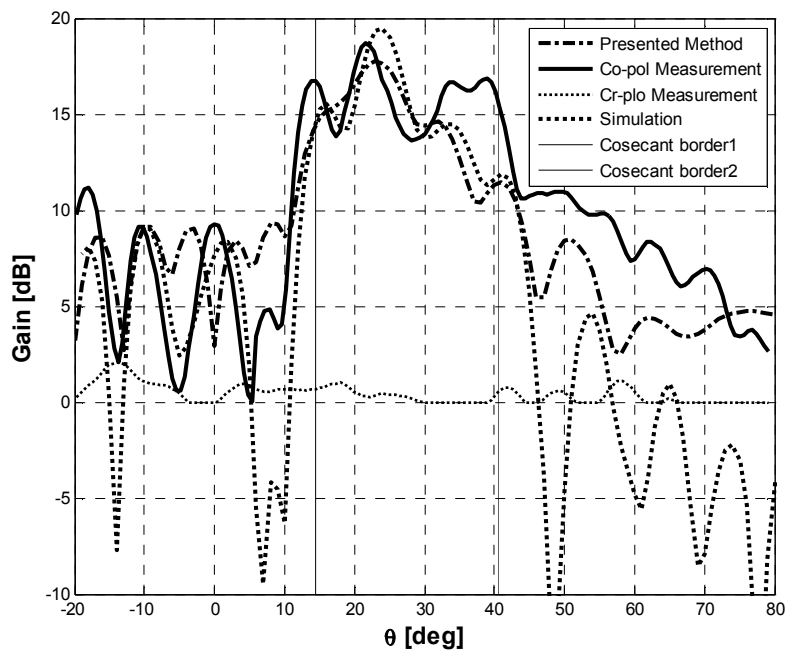

Figure 9. Comparison between measured, simulated and theoretical radiation patterns at $9.5 \mathrm{GHz}$.

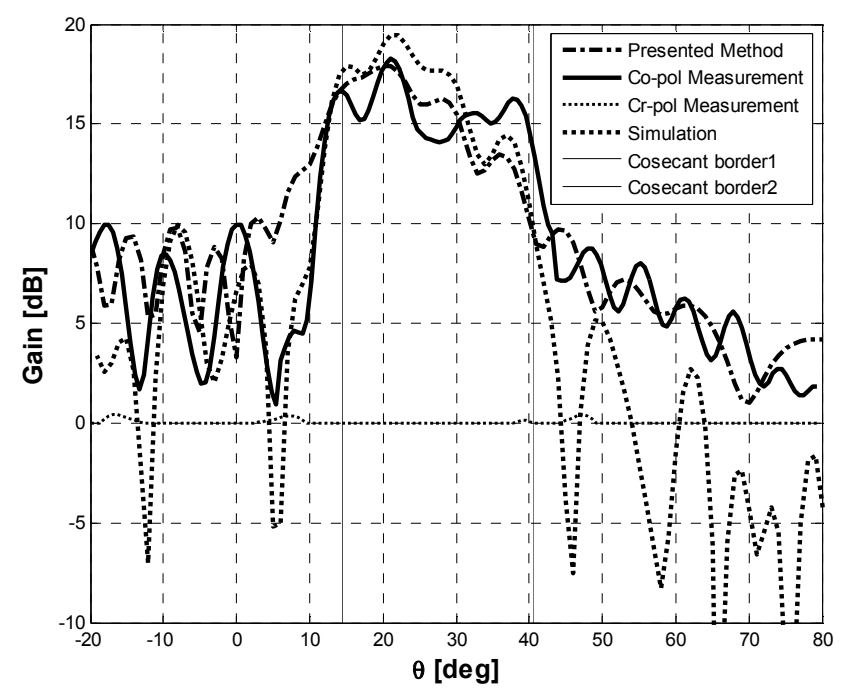

Figure 10. Comparison between measured, simulated and theoretical radiation patterns at $10.5 \mathrm{GHz}$. 


\section{Conclusion}

A new transmit array structure was presented, which consist of a nonuniform dielectric layer with lattice of uniform elements and a feed-horn antenna. Nonuniform dielectric layer with variable thickness distribution was used to control the transmission phase. In the analyzing section, an efficient method was implemented for designing and analyzing such transmit array antenna. This method is based on equivalent transmission line model, which has the distinct advantages of simplicity and rapidity in analyzing and designing of such antennas. Then, after designing and finding the proper value of thickness for each element, the shaped beam transmit array antenna was obtained. Finally, the performance of proposed antenna was verified through presenting an example at $10 \mathrm{GHz}$ frequency. The shaped beam transmit array antenna has been manufactured and tested in an anechoic chamber showing a good agreement between the theoretical, simulated and the measured radiation patterns. The measurement results showed that for the $30 \mathrm{~cm}$ square nonuniform transmit array antenna, the squared cosecant pattern in the range of $9.5-10.5 \mathrm{GHz}$ was obtained.

\section{References}

[1] D. M. Pozar, "Flat lens antenna concept using aperture coupled microstrip patches," Electronics Letters, vol. 32, no. 23, pp. 2109-2111, Nov. 1996.

[2] W. C. Choi, S. Lim and Y. J. Yoon, "Design of Noninvasive Hyperthermia System Using Transmit-Array Lens Antenna Configuration", IEEE Antennas and Wireless Propagation Letters, Vol. 15, pp. 857-860, 2016.

[3] D. Popovic and Z. Popovic, "Multibeam antenna with polarization and diversity," IEEE Trans. Antennas Propag., Vol. 50, No. 5, pp. 651-657, May 2002.

[4] J. Y. Lau, S. V. Hum, "A wideband reconfigurable transmitarray element," IEEE Trans. Antennas Propag., Vol. 60, No. 3, pp. 1303-1311, Mar. 2012.
[5] A. D. Nemshon, A. M. Alexandrin, S. V. Polenga, A. V. Stankovsky, V. S. Panko and Y. P. Salomatov, "A Broadband Sub-Wavelength Phase-Correcting Element for Transmit Antenna Arrays", 24th Int. Crimean Conference Microwave \& Telecommunication Technology" (CriMiCo'2014), pp. 469470, 7-13 September, Sevastopol, Crimea, Russia, 2014.

[6] Y. Takahashi, N. Honma and Y. Suzuki, "Using a Tunable Transmit-Array Antenna to Improve the Propagation Environment", IEEE Antennas and Wireless Propagation Letters, Vol. 12, pp. 825-827, 2013.

[7] I. V. Minin, O. V. Minin, "Basic principles of Fresnel Antenna Arrays", Springer, 2010.

[8] I. V. Minin, O. V. Minin, A. Petosa and S. Thirakoune, "Improved Zoning Rule for Designing Square Fresnel Zone Plate Lenses", Microwave and Optical technology Letters, Vol. 49, No. 2, pp. 276278, 2007.

[9] Y. Zhang, R. Mittra and W. Hong, "A Zoned Two-Layer Flat Lens Design", Int. Workshop on Antenna Technology (IWAT), pp. 412-415, 2011.

[10] D. M. Pozar and S. D. Targonski, "Design of millimeter wave microstrip reflectarrays," IEEE Trans. Antennas Propag., Vol. 45, No. 2, pp. 287-295, Feb. 1997.

[11] M. Moeini-Fard and M. Khalaj-Amirhosseini, "Nonuniform Reflect-Array Antennas", Int. Journal of RF and Microwave Computer-Aided Engineering, Vol. 22, No. 5, pp. 575-580, Sep. 2012.

[12] Z. Hamzavi-Zarghani and Z. Atlasbaf, "A New Broadband Single-Layer Dual-Band Reflectarray Antenna in X- and $\mathrm{Ku}-$ Bands", IEEE Antennas and Wireless Propagation Letters, Vol. 14, pp. 602-605, 2015.

[13] R. H. Clark, J. Brown, Diffraction theory and antennas, Ellis Horwood, Chichester 1980.

[14] A. Chakraborty, B. N. Das, and G. S. Sanyal, "Beam shaping using nonlinear phase distribution in a uniformly spaced array", IEEE Trans. Antennas Propag., Vol. 30, pp. 10311034, Sep. 1982. 